\title{
Telemetric hemodynamic assessment during anesthesia induction with propofol in experimental heart failure
}

\author{
Julia Vogel $^{1-3}$, Philip Boehme ${ }^{1,2}$, Reza Wakili ${ }^{3}$, Katharina Boden ${ }^{2}$, Thomas Reimer ${ }^{1}$, Peter Sandner ${ }^{1,5}$, Jörg Hüser $^{1}$, Wilfried Dinh ${ }^{1,2,4}$, Hubert \\ Truebel $^{1,2}$ and Thomas Mondritzki ${ }^{1,2 *}$ \\ ${ }^{1}$ Bayer AG, Wuppertal, Germany \\ ${ }^{2}$ University of Witten/Herdecke, Witten, Germany \\ ${ }^{3}$ Department of Cardiology and Vascular Medicine, West German Heart and Vascular Center Essen, University of Essen Medical School, University Duisburg- \\ Essen, Essen, Germany \\ ${ }^{4}$ Department of Cardiology, HELIOS Clinic Wuppertal, University Hospital Witten/Herdecke, Wuppertal, Germany \\ ${ }^{5}$ Hannover Medical School, Hannover, Germany
}

\begin{abstract}
Background: The influence of initial propofol on cardiac hemodynamics in patients suffering from heart failure (HF) is insufficiently understood and contradicting opinions are existing. Nevertheless, propofol is often used in HF patients if etomidate is contraindicated. The development of novel telemetry sensors allows the assessment of intracardiac pressure signals in conscious animals and thus the investigation of anesthesia induction. Here, we investigated the hemodynamic effects of propofol in healthy dogs in comparison to dogs affected with HF.

Methods: Telemetry sensors and pacemaker were implanted in six male beagle dogs. After wound healing, the pacemaker was programmed to 220 bpm and later to $180 \mathrm{bpm}$ to induce and maintain heart failure. At baseline conditions (before pacing) and at several time points (Day 14,28 and 42) during HF progression all animals were anesthetized with $5 \mathrm{mg} / \mathrm{kg}$ propofol i.v. Hemodynamic measurements were done to assess the effects of propofol during anesthesia induction

Results: After induction with propofol, we observed a significant ( $<<0.001$ ) increase in heart rate (HR) for all groups (healthy: $+63 \pm 53 \mathrm{bpm}, 14 \mathrm{~d}$ : $+48 \pm 23 \mathrm{bpm}, 28 \mathrm{~d}$ : $+45 \pm 20 \mathrm{bpm}, 42 \mathrm{~d}:+51 \pm 28 \mathrm{bpm}$ in peak) compared to individual baseline values. In addition, blood pressure (BP) increased in all HF groups (in average $+17.4 \pm$ $1.4 \mathrm{mmHg}, \mathrm{p}<0.05)$, while this effect was not present under healthy conditions. We further observed a trend towards an increase in left ventricular contractility (+dP/ dtmax) by $+621.1 \pm 157.0 \mathrm{mmHg} / \mathrm{s}$ (averaged peak data) in all groups.

Conclusions: In dogs affected with heart failure, initial anesthesia with propofol resulted in an increase in heart rate and blood pressure, whereas no cardio depressive adverse effects could be observed on HF-relevant parameters. Therefore, propofol might be an alternative for short term anesthesia in HF patients. Nevertheless, further animal and human studies are needed to evaluate if these data can be translated into the clinical setting.
\end{abstract}

\begin{abstract}
Abbreviations: BNP: Brain natriuretic peptide; CVD: Cardiovascular disease; DBP: Diastolic blood pressure; GABAAR: Gamma aminobutyric acid A receptor; HF: Heart failure; HFrEF: Heart failure with reduced ejection fraction; HR: Heart rate; LVEDP: Left ventricular end diastolic pressure; LVP: Left ventricular pressure; MBP: Mean blood pressure; mmHg: Millimeters mercury; MRT: Magnetic resonance tomography; SBP: Systolic blood pressure; SD: Standard deviation; SEM: Standard error of the mean; BW: Body weight; Ntpro BNP: $\mathrm{N}$ terminal brain natriuretic peptide; EF: Ejection fraction; LVEDDV A4C: Left ventricular enddiastolic volume measured in four chamber view; LVLd A4C: Left ventricular length at diastole measured in four chamber view; LVESV A4C: Left ventricular endsystolic volume measured in four chamber view; LVLs A4C: Left ventricular lenght at systole measured in four chamber view; LVEDDV A2C: Left ventricular enddiastolic volume measured in two chamber view; LVLd A2C: Left ventricular length at diastole measured in two chamber view; LVESV A2C: Left ventricular endsystolic volume measured in two chamber view; LVLs A2C: Left ventricular lenght at systole measured in two chamber view; LVEDP: Left ventricular enddiastolic volume; HR: Heart rate; MBP: Mean blood pressure; +dP/dt: Contractility; -dP/ dt: Relaxation; Tau: Relaxation-time-constant.
\end{abstract}

\section{Introduction}

With almost 26 million deaths in 2015, cardiovascular diseases (CVD) are the main cause of mortality worldwide [1]. It is expected that this number will further rise, reflecting not only an aging global population but also the consequences of the growing prevalence of obesity and metabolic syndrome. This CV burden is also associated with a rise in cardiac and non-cardiac surgical interventions $[2,3]$.

In comparison to healthy subjects patients with CVD have an impaired cardiac function that may be further compromised by anesthesia [2]. It is important to avoid a decrease in myocardial contractility, to maintain normal blood pressure and coronary perfusion and to prevent an afterload increase in predisposing patients for arrhythmias and thromboembolic events $[3,4]$.

${ }^{*}$ Correspondence to: Thomas Mondritzki, Bayer AG, BAG-DD-TRG-CV III Building 500, D-42096 Wuppertal, Germany, E-mail: thomas.mondritzki@ bayer.com

Key words: anesthesia, propofol, heart failure, animal study, telemetry

Received: July 09, 2020; Accepted: July 21, 2020; Published: July 24, 2020 
Propofol, a dialkylphenol- $\gamma$-aminobutyric-acid-A-receptor (GABAAR) agonist, is a widely applied agent for intravenous (i.v.) anesthesia, which is used for induction and for anesthesia maintenance. The advantages are based on its fast effect-onset and short half-life. However, the use in patients with HF is viewed unfavorably by some authors, because of its potential vasodilatory and BP-lowering effects $[5,6]$. Only a few studies investigated the effect of continuous infusion of propofol in patients and animals with left ventricular dysfunction [79]. Interestingly, most of these studies did not consider the induction phase due to the inability for continuous monitoring of intra-cardiac hemodynamics in non-sedated patients. Most studies did only investigate electrocardiogram (ECG) and BP [10-12]. Only one study examined intra-cardiac hemodynamics during induction in conscious, healthy sheep where initial propofol led to negative inotropic and positive chronotropic effects [13].

It can be assumed that the period of anesthesia initiation is the most vulnerable phase and should critically be considered. Due to highest plasma concentration in this initial phase, strongest hemodynamic changes can be expected. Previous research methods (plasma biomarker, echocardiography, Magnetic Resonance Tomography (MRT) or right-/left heart catheterization), especially in animal models had certain limitations requiring prior sedation thereby not allowing a real assessment in the anesthesia induction phase. The development of telemetry-systems can help to overcome this knowledge gap. Newly developed implantable sensors are able to continuously monitor high resolution hemodynamic data in conscious animals in absence of any sedation $[14,15]$.

These 'digital biomarkers' could provide an option to assess the systemic and cardiac hemodynamic situation during initial anesthesia.

In this study we aimed to investigate the safety of anesthesia initiation with propofol in dogs at healthy status and after tachypacing induced HF by the use of implantable telemetry devices with respect to intra-cardiac hemodynamic parameters.

\section{Methods}

\section{Ethics statement}

All study procedures complied with the current national legislation (German protection of animals act [May 18, 2016], last amended by article 4 paragraph 87 [BGBl. I S. 1666] on July 18, 2016, and the EU directives 63/2010 [on the protection of animals used for scientific purposes]). All study protocols were approved by the competent regional regulatory authority (LANUV NRW in Duesseldorf/ Germany) and by the institutional animal care office of Bayer AG. The investigation conforms to the Guide for the Care and Use of Laboratory
Animals published by the US National Institutes of Health (publication no. 85-23, revised 1985). This study was part of a larger study which aimed to investigate new drug candidates.

\section{Study protocol}

The study protocol is shown in Figure 1. Six male Beagle dogs (Marshall, BioResources), weighing between 10 and $14 \mathrm{~kg}$, underwent surgical interventions for telemetric sensor and pacemaker implantation as described below. After wound healing, all animals studied in the conscious state during spontaneous heart rhythm. On day 0 , pacemakers were activated (Mode: A00, Current: 3.6V, Frequency: 220 beats per minute) and continued for 28 days to induce HF. After 28 days of pacing at a rate of 220 beats per minute the pacing rate was reduced to 180 beats per minute for 14 days (Mode: A00, Current: $3.6 \mathrm{~V}$, Frequency: 180 beats per minute) to achieve stable HF conditions without further progression. To investigate the hemodynamic effect of propofol, acute examinations took place at baseline (14 days before tachypacing, representing healthy status) and after 14, 28 and 42 pacing. For HF assessment, echocardiography, soluble biomarker (NTproBNP) analysis and telemetric hemodynamic measurements were performed.

\section{Surgical interventions}

For both (telemetry sensor and pacemaker) implantations, all dogs were initially anesthetized with thiopental-Na $(0.25-0.5 \mathrm{~g} /$ animal Trapanal ${ }^{\circ}$, Byk Gulden, Germany) and pancuroniumbromid (0.2 - $0.25 \mathrm{mg} / \mathrm{kg}$ Pancuronium Inresa, Inresa Arzneimittel GmbH, Germany) for muscle relaxation. The anesthesia supplementation was administered via the anesthetic gas isoflurane (1-2 \%, Isofluran Baxter, Baxter, Germany). Fentanyl (10 - $40 \mu \mathrm{g} / \mathrm{kg} / \mathrm{h}$ Fentanyl, Mallinckrodt Inc, United Kingdom) was infused through the right cephalic vein for analgesia. During implantation all animals were intubated and mechanically ventilated with $30 \%$ oxygen using Avance $\odot$ anesthesia ventilator (GE Healthcare, Germany). For fluid substitution $3 \mathrm{ml} / \mathrm{kg}$ physiologic solution ( $\mathrm{NaCl} 0.9 \%$, B. Braun, Germany) were applied via infusion for the duration of the surgery.

A telemetry sensor (Model-no.: L21, Data Sciences International, USA) was inserted via left-sided thoracotomy and remained implanted in these animals to monitor hemodynamic parameters. Those parameters are: ECG, blood pressure (BP), left ventricular pressure (LVP), locomotor activity and body temperature. Two ECG leads were fixed in an apical and basal position of the heart. One pressure catheter was placed in the thoracic aorta (BP) and a second pressure catheter was placed in the left ventricle through the apex of the heart. The ECG and both pressure catheters were connected with the electronic housing of the device, which was placed in the submuscular space on the left thorax side [16].

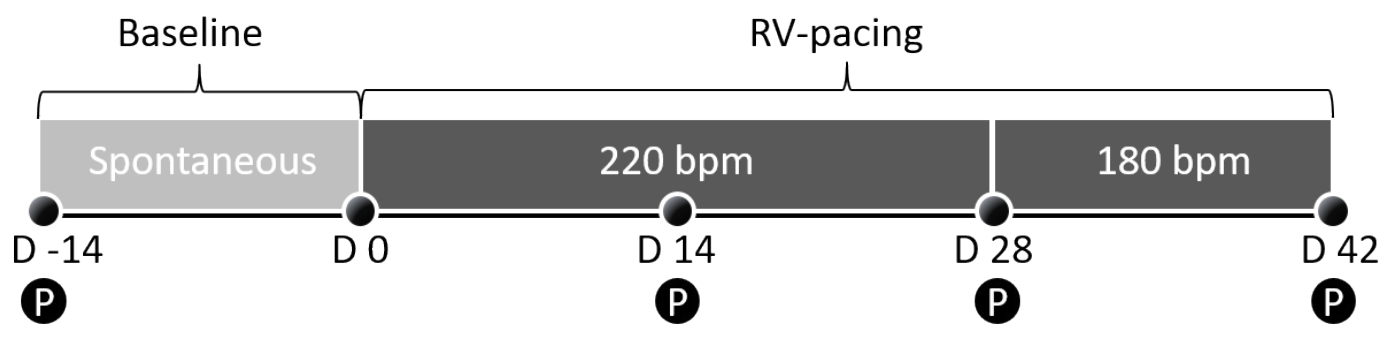

Figure 1. Overall study protocol ( $\mathrm{P}=\mathrm{Propofol}$ testing) 
A pacemaker lead (Model-no.: Setrox S53, Biotronik, Germany) was inserted via the right jugular vein into the right ventricle. This lead was connected with the aggregate of the pacemaker and was placed subcutaneously between the shoulder blades.

All devices were checked for adequate and plausible signal quality before wound closure. All dogs received antibiotic $(12.5 \mathrm{mg} / \mathrm{kg}$, Clindamycin po (Clerobe $\left.{ }^{\odot}\right)$, Zoetis, Germany) and analgesic $(4 \mathrm{mg} / \mathrm{kg}$ Carprofen po (Rimadyl ${ }^{\circ}$ ), Pfizer, USA) treatment over a period of 10 days after implantation. For additional analgesia a Durogesic ${ }^{\circ}$ patch ( $25 \mu \mathrm{g} / \mathrm{h}$, Fentanyl, Janssen-Cilag, Germany) was placed on the dorsal site for three days after surgery.

HF assessment by echocardiography, NT-proBNP and telemetric hemodynamic assessment

Transthoracic echocardiography was performed in conscious animals at days $-14,14,28$ and 42 to determine heart dimensions and ejection fraction from biplane 2 and 4 chamber views (Vivid I, GE Healthcare, USA). All analyses were done using the software EchoPAC ${ }^{\mathrm{m}}$ (GE Healthcare, USA).

Blood samples were collected on weekly basis to determine plasma NT-proBNP using a Cardiopet proBNP Elisa test (IDEXX Laboratories, Germany). All blood samples were stored at $-20^{\circ} \mathrm{C}$ (after centrifugation at $3500 \mathrm{rpm}$ for $10 \mathrm{~min}$ ), until analyses were performed at IDEXX laboratories.

To evaluate the baseline systemic and cardiac performance during healthy and HF conditions, hemodynamic measurements were conducted on day $-14,14,28$ and 42 while the pacemaker was switched off for a short time. Telemetric data were then obtained and analyzed immediately before anesthesia initiation with propofol for a period of 2 minutes.

\section{Hemodynamic assessment during propofol anesthesia}

The advantage of an implanted telemetric sensor is the possibility to continuous monitor hemodynamic parameters, independent of the consciousness state.

On day $-14,14,28$ and 42 (Figure 1) all dogs were investigated for their response to intravenous propofol. The acute study protocol is shown in Figure 2. Animals received an intravenous access and after a short recovery period a baseline hemodynamic recording of two minutes started as described above and was used as reference. Afterwards, a propofol bolus $(5 \mathrm{mg} / \mathrm{kg}$, Propofol, Fresenius Kabi, Germany) was given at a constant rate of 30 seconds per injection. Another three minutes of data recording was performed to evaluate the effect of propofol on cardiac and systemic hemodynamics. Afterwards, the animals recovered from anesthesia and pacemakers were reprogrammed into pacing mode.

\section{Statistics}

The transmitted signals were collected by telemetry receivers (Model TRX-1, Data Sciences International, USA) and were transferred to a computer and processed by the Ponemah P3 Plus software (V. 5.2 service pack 9, Data Sciences International, USA). ECG and pressure data were averaged over a predefined period ("logging rate") of 10 seconds. From these recordings, the systolic (SAP), diastolic (DAP) and mean blood pressure (MAP) were derived. The heart rate is expressed in beats per minute (bpm) and was derived from the ECG (RR-Intervals for the cardiac cycle multiplied by 60 ). From the phasic left ventricular pressure (LVP) signal, the maximum positive $(+\mathrm{dP} / \mathrm{dt})$ and negative $(-\mathrm{dP} / \mathrm{dt})$ values of the first derivative occurring during the cardiac cycle were analyzed. $+\mathrm{dP} / \mathrm{dt}$ is a common, robust and sensitive indicator of changes in cardiac contractility, whereas - $\mathrm{dP} / \mathrm{dt}$ indicates changes in relaxation. Furthermore, the parameter of left ventricular enddiastolic pressure (LVEDP, pressure at the last zero crossing of the differentiated pressure during the rise to the systolic period) and the time constant isovolumic left ventricular pressure decay (TAU) was analyzed from the LVP-signal. Data could be evaluated online or be stored and evaluated offline.

For creations of graphics and for statistical analysis all data were processed by GraphPad Prism ${ }^{\circ}$ software (Version 7.0, GraphPad Software Inc., USA). Data was expressed as mean +/- standard error of the mean (SEM) in graphics or standard deviation (SD). A repeated measures one-way ANOVA with Geisser-Greenhouse correction and Dunnett's multiple comparison test was performed to compare group differences in model characteristics on individual days of examination. The statistically analysis of propofol-related effects based on a repeated measures one-way ANOVA test followed by a Dunnett's multiple comparison test. Therefore, all data collected in the pre-application phase (-120 sec - $0 \mathrm{sec}$ pre application of Propofol) were averaged and compared to individual data after application. Significant differences to baseline values were marked in the figures and the lowest level of significance highlighted. An $\alpha$ - level of $\mathrm{p}<0.05$ for statistical significance was chosen.

\section{Results}

\section{Heart failure model}

This study aimed to investigate the cardiovascular effects during anesthesia induction using propofol in HF versus healthy subjects. Therefore, dogs were instrumented with telemetry sensors and pacemakers were implanted. The implantation of pacemaker and telemetry devices were well tolerated in all animals and all sensors delivered accurate signals during the course of the study. Furthermore, all pacemaker leads were in a right position and specifically no dislocation could be observed.

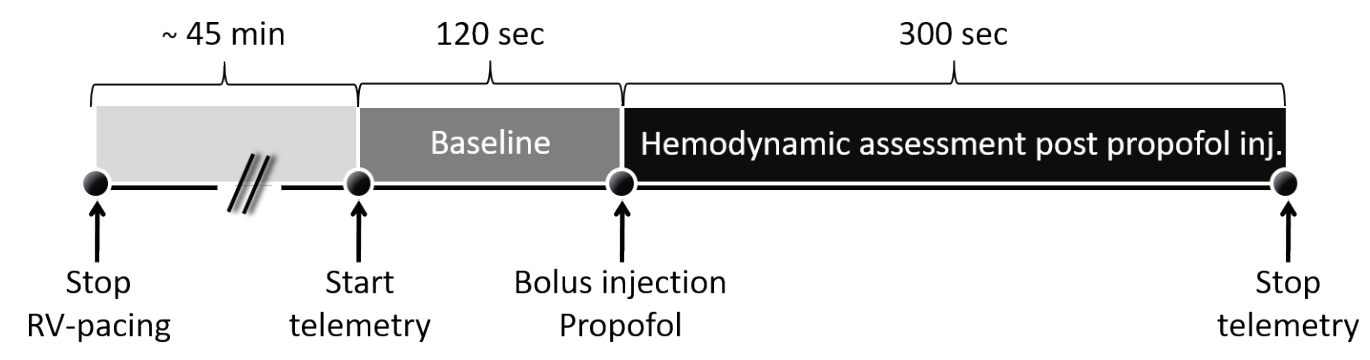

Figure 2. Acute study protocol performed on day -14, 14, 28 and 42 


\section{Baseline characteristics}

Table 1 shows the model characteristics before (day -14) and after (day 14, 28, 42) HF induction. It could be demonstrated that tachypacing led to a significant increase in the plasma Biomarker NTproBNP from $437 \pm 150 \mathrm{pmol} / \mathrm{l}$ to $4891 \pm 1510 \mathrm{pmol} / \mathrm{l}(\mathrm{p}=0.0010)$ after 14 days of pacing. This effect was more pronounced after 28 days (5641 $\pm 1576 \mathrm{pmol} / \mathrm{l}, \mathrm{p}=0.0007$ ), which concurs with the further progression of HF [17]. After 42 days of pacing, no examinations of the plasma samples were performed. Similar to literature data, echocardiographic assessment shows a robust and significant decrease in ejection fraction (14d of pacing: $-26.1 \pm 4.0 \%$; $28 \mathrm{~d}$ of pacing: $-29.1 \pm 4.2 \%$; $42 \mathrm{~d}$ of pacing: $-28.0 \pm 3.7 \%$, all $\mathrm{p}<0.0001)$ and increase in LV endsystolic $(+15.2 \pm 2.5$ $\mathrm{ml}, 28 \mathrm{~d}$ of pacing, $\mathrm{p}<0.0001)$ and enddiastolic $(+11.9 \pm 2.7 \mathrm{ml}, 28 \mathrm{~d}$ of pacing, $\mathrm{p}<0.0001$ ) volumes.

The implanted telemetric system delivers cardiac hemodynamic parameters continuously during the time course of the study. Provoked by a tachycardia-induced heart failure, we observed changes in the following systemic and cardiac parameters: Analyzing the heart rate, a statistically significant increase after 14 days $(+21.3 \pm 12.2 \mathrm{bpm}$, $\mathrm{p}=0.0185), 28$ days $(+31.2 \pm 20.7 \mathrm{bpm}, \mathrm{p}=0.0329)$ and 42 days $(+20.5 \pm$ $12.3 \mathrm{bpm}, \mathrm{p}=0.0227$ ) of tachypacing was observed. Mean arterial blood pressure showed a statistically non-significant decrease after 14 days $(-10.2 \pm 7.9 \mathrm{mmHg}, \mathrm{p}=0.0987)$ and no effects after 28 days $(-3.8 \pm 18.7$ $\mathrm{mmHg}, \mathrm{p}=0.9370)$ and 42 days $(-2.7 \pm 5.6 \mathrm{mmHg}, \mathrm{p}=0.6235)$.

There was a slight, statistically non-significant trend, for an increase in LVEDP after 28 days $(+5.1 \pm 5.7 \mathrm{mmHg}, \mathrm{p}=0.1736)$, and no effects after $14(+0.1 \pm 4.5 \mathrm{mmHg}, \mathrm{p}>0.999)$ and 42 days $(+2.2 \pm 6.0 \mathrm{mmHg}$, $\mathrm{p}=0.7211)$ of pacing. In contractility, there was a robust and significant decrease after 14 days $(-2398.5 \pm 1076.7 \mathrm{mmHg} / \mathrm{s}, \mathrm{p}=0.0067), 28$ days $(-2154.9 \pm 952.9 \mathrm{mmHg} / \mathrm{s}, \mathrm{p}=0.0063)$ and 42 days $(-2119.6+/-890.0$ $\mathrm{mmHg} / \mathrm{s}, \mathrm{p}=0.0050)$. Also, relaxation showed a statistically significant decrease after 14 days $(-2021.3 \pm 457.0 \mathrm{mmHg} / \mathrm{s}, \mathrm{p}=0.0003), 28$ days
$(-2095.6 \pm 583.9 \mathrm{mmHg} / \mathrm{s}, \mathrm{p}=0.0008)$ and 42 days $(-1814.1+/-554.3$ $\mathrm{mmHg} / \mathrm{s}, \mathrm{p}=0.0012)$. Tau, a marker of diastolic function, significant increased after 14 days $((+17.9 \pm 9.5 \mathrm{msec}, \mathrm{p}=0.0236)$ and 28 days $(+17.9 \pm 9.5 \mathrm{msec}, \mathrm{p}=0.0396)$ of pacing and showed no significant effect after 42 days $(+13.7+/-14.3 \mathrm{msec}, \mathrm{p}=0.0798)$. From these baseline characteristics it can be assumed that all animals have developed stable heart failure.

\section{Hemodynamics during anesthesia induction}

In Figure 3 continuous hemodynamic recordings are shown during anesthesia induction with propofol. Displayed are the individual hemodynamic parameters during anesthesia initiation, starting at 120 seconds before i.v.-bolus application of propofol and ending up 180 seconds after start of propofol administration. To better quantify the effect of propofol in comparison to baseline conditions (before propofol) and to visualize effects associated with HF progression, all data were illustrated as changes to individual averaged baseline values (Figure 4). For this purpose, the data were averaged before application $(120 \mathrm{sec}-0 \mathrm{sec})$ and compared to individual values collected after application $(0 \mathrm{sec}-180 \mathrm{sec})$.

As shown in Figure $3 \mathrm{~A}$ and $4 \mathrm{~A}$, we could demonstrate that anesthesia induction with propofol led to a substantial and significant increase in heart rate (healthy: $+69.2 \pm 71.4 \%, \mathrm{p}<0.0001 ; 14 \mathrm{~d}:+39.6 \pm$ $20.4 \%, \mathrm{p}<0.0001 ; 28 \mathrm{~d}:+34.7 \pm 16.0 \%, \mathrm{p}=0.0006 ; 42 \mathrm{~d}:+43.2 \pm 26.6 \%$, $\mathrm{p}<0.0001$ in peak, $\mathrm{p}<0.0001$ ) in all groups without any relevant group differences. The peak effect within the observation period of $180 \mathrm{sec}$ was shown between 60 and $100 \mathrm{sec}$ post start application. Figure 4A shows, that the largest increase in heart rate, compared to corresponding baseline conditions, was observed in healthy animals with $63.2 \pm 53.4$ beats per minute ( $80 \mathrm{sec}$ post appl.). The maximum absolute deflection was reached with $177.0 \pm 13.8$ beats per minute after 28 days of pacing (100 sec post appl.). Overall, there was no more or less pronounced effect in heart rate observable between healthy animals and dogs affected with HF.

Table 1. Model characteristics at individual days of examination

\begin{tabular}{|c|c|c|c|c|}
\hline & Healthy & & Heart failure & \\
\hline Characteristic & & 14 days of pacing & 28 days of pacing & 42 days of pacing \\
\hline No. of animals & 6 & 6 & 6 & 6 \\
\hline $\mathrm{BW}[\mathrm{kg}]$ & $11.6 \pm 1.6$ & $11.6 \pm 1.6$ & $11.6 \pm 1.7$ & $11.6 \pm 1.8$ \\
\hline \multicolumn{5}{|l|}{ Biomarker } \\
\hline Nt-pro BNP [pmol/1] & $437 \pm 150$ & $48917 \pm 1510 * * *$ & $5641 \pm 1576^{* * *}$ & \\
\hline \multicolumn{5}{|l|}{ Echocardiography } \\
\hline $\mathrm{EF}[\%]$ & $58.3 \pm 5.0$ & $32.3 \pm 3.2 * * * *$ & $29.3 \pm 2.3 * * * *$ & $30.3 \pm 2.2 * * * *$ \\
\hline LVEDV A4C [ml] & $23.8 \pm 4.0$ & $30.2 \pm 8.4$ & $34.7 \pm 6.3^{* *}$ & $33.6 \pm 4.2 * * *$ \\
\hline LVLd A4C [cm] & $4.4 \pm 0.4$ & $4.8 \pm 0.3$ & $4.9 \pm 0.3$ & $5.0 \pm 0.3^{* *}$ \\
\hline LVESV A4C [ml] & $10.3 \pm 2.7$ & $20.6 \pm 6.4^{* *}$ & $24.4 \pm 5.2 * * *$ & $23.3 \pm 3.7 * * *$ \\
\hline LVLs A4C [cm] & $3.6 \pm 0.4$ & $4.3 \pm 0.4^{* *}$ & $4.5 \pm 0.3 * *$ & $4.4 \pm 0.4^{* * *}$ \\
\hline LVEDV A2C [ml] & $24.8 \pm 2.1$ & $30.9 \pm 5.6^{*}$ & $37.7 \pm 3.8 * * *$ & $38.6 \pm 7.6^{*}$ \\
\hline LVLd A2C [cm] & $4.6 \pm 0.4$ & $5.0 \pm 0.3^{*}$ & $5.2 \pm 0.4 * *$ & $5.2 \pm 0.4 * *$ \\
\hline LVESV A2C [ml] & $10.3 \pm 1.8$ & $20.9 \pm 4.8^{* *}$ & $26.6 \pm 3.7 * * *$ & $26.6 \pm 5.9^{* *}$ \\
\hline LVLs A2C [cm] & $3.8 \pm 0.5$ & $4.4 \pm 0.3^{* *}$ & $4.7 \pm 0.3 * *$ & $4.7 \pm 0.4^{* *}$ \\
\hline \multicolumn{5}{|l|}{ Hemodynamics } \\
\hline LVEDP $[\mathrm{mmHg}]$ & $12.1 \pm 3.3$ & $12.1 \pm 3.6$ & $17.2 \pm 6.3$ & $14.2 \pm 6.2$ \\
\hline HR [bpm] & $100.8 \pm 15.5$ & $122.2 \pm 4.1^{*}$ & $132.0 \pm 7.8^{*}$ & $121.3 \pm 9.4^{*}$ \\
\hline $\mathrm{MBP}^{\#}[\mathrm{mmHg}]$ & $84.7 \pm 3.5$ & $74.5 \pm 8.2$ & $80.9 \pm 19.5$ & $82.0 \pm 6.1$ \\
\hline$+\mathrm{dP} / \mathrm{dt}[\mathrm{mmHg} / \mathrm{s}]$ & $4285 \pm 1199$ & $1887 \pm 153 * *$ & $2130 \pm 303^{* *}$ & $2165 \pm 358^{* *}$ \\
\hline$-\mathrm{dP} / \mathrm{dt}[\mathrm{mmHg} / \mathrm{s}]$ & $4266 \pm 600$ & $2245 \pm 298^{* * * *}$ & $2170 \pm 245^{* * *}$ & $2452 \pm 431 * *$ \\
\hline Tau $[\mathrm{msec}]$ & $15.8 \pm 4.7$ & $34.3 \pm 7.3^{*}$ & $30.1 \pm 11.3 *$ & $26.8 \pm 9.7$ \\
\hline
\end{tabular}

Data are expressed as mean of $\mathrm{n}=6 \pm \mathrm{SD}$. $* \mathrm{p}<0.01 ; * \mathrm{p}<0.001 ; * * * \mathrm{p}<0.0001 ; * * * \mathrm{p}<0.00001$. Repeated measurements one-way ANOVA with Greenhouse correction. A Dunnett test was used for multiple comparison. A mixed-effects analysis was done in case of missing values. \#: MBP was measured in 5 dogs. 
A similar effect could be observed for blood pressure (Figure 3 B-D, Figure 4 B-D). With the exception of the time point when the animals were healthy, all measured pressures (systolic, mean and diastolic pressure) significantly $(\mathrm{p}<0.05)$ increased between $130-150 \mathrm{sec}$ post application of propofol. In the three minutes observational period post application, the maximum rise in mean blood pressure was $+19.5 \pm$ $13.6 \mathrm{mmHg}(\mathrm{p}=0.0013)$ after 14 days of pacing, $+17.1 \pm 14.3 \mathrm{mmHg}$ $(\mathrm{p}=0.0205)$ after 28 days of pacing and $+17.3 \pm 11.3 \mathrm{mmHg}(\mathrm{p}=0.0089)$ after 42 days of pacing. In contrast, in healthy animals we observed a non-significant increase in mean blood pressure of $8.7 \pm 20.9 \mathrm{mmHg}$ in maximum.
In contractility (Figure 3E, 4E) no further negative-inotropic effects induced by propofol was observed for any time point in HF animals. At all times at most a slight (healthy: $+819.0 \pm 2085.7 \mathrm{mmHg} / \mathrm{s} ; 28 \mathrm{~d}$ : $+674.1 \pm 764.7 \mathrm{mmHg} / \mathrm{s} ; 42 \mathrm{~d}:+511.7 \pm 690.4 \mathrm{mmHg} / \mathrm{s}$ in peak) increase in $+\mathrm{dP} / \mathrm{dt}$ could be observed, which was signifcant at a single timepoint $(+479.7 \pm 560.5 \mathrm{mmHg} / \mathrm{s}, 100 \mathrm{sec}$ post application, $\mathrm{p}=0.0099)$ after 14 days of pacing. In contrast, no effect was observed for the parameter 'Relaxation' as shown in Figure 3F and 4F.

Left ventricular enddiastolic pressure (Figure 3G, 4G), a marker of preload of the heart was not significantly affected by propofol injection. However, a trend towards higher values was observed and

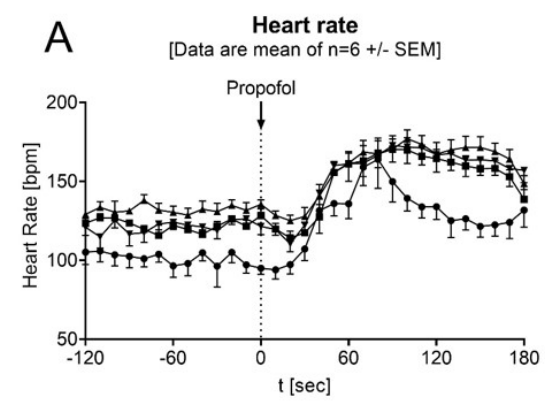

B
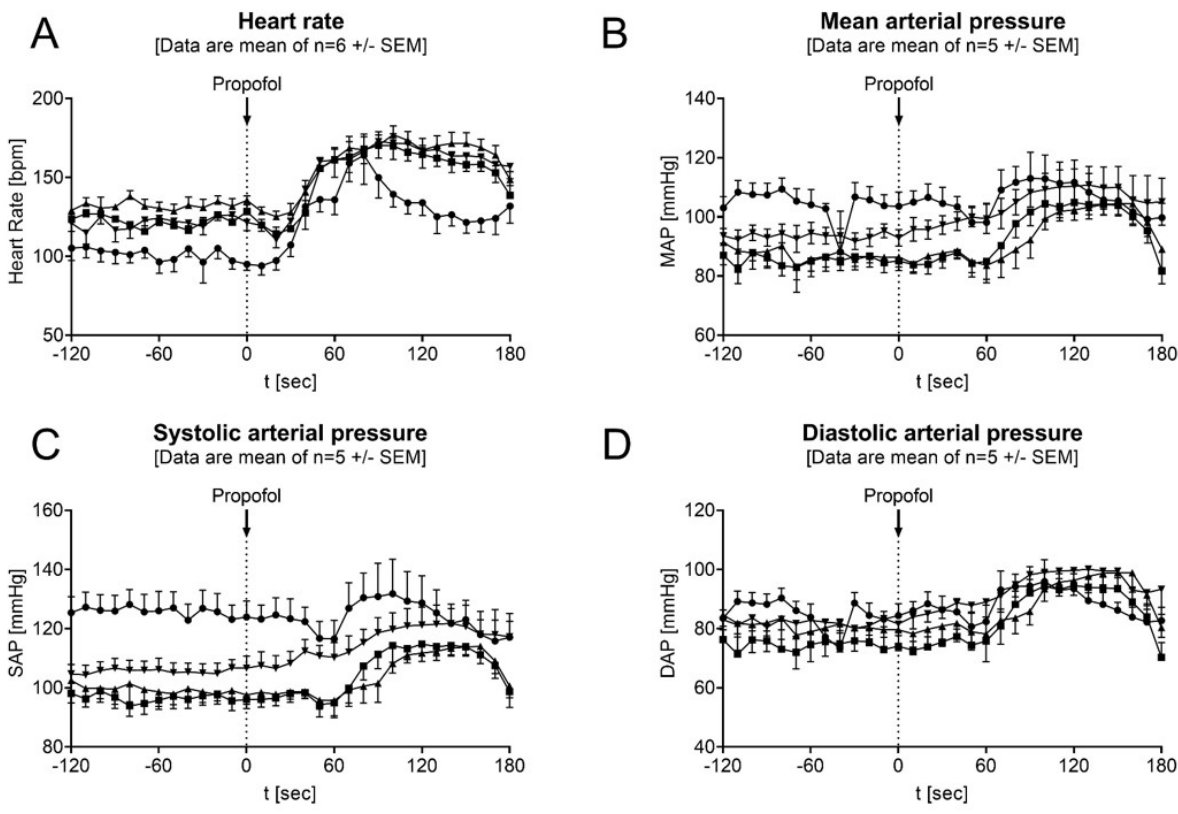

D
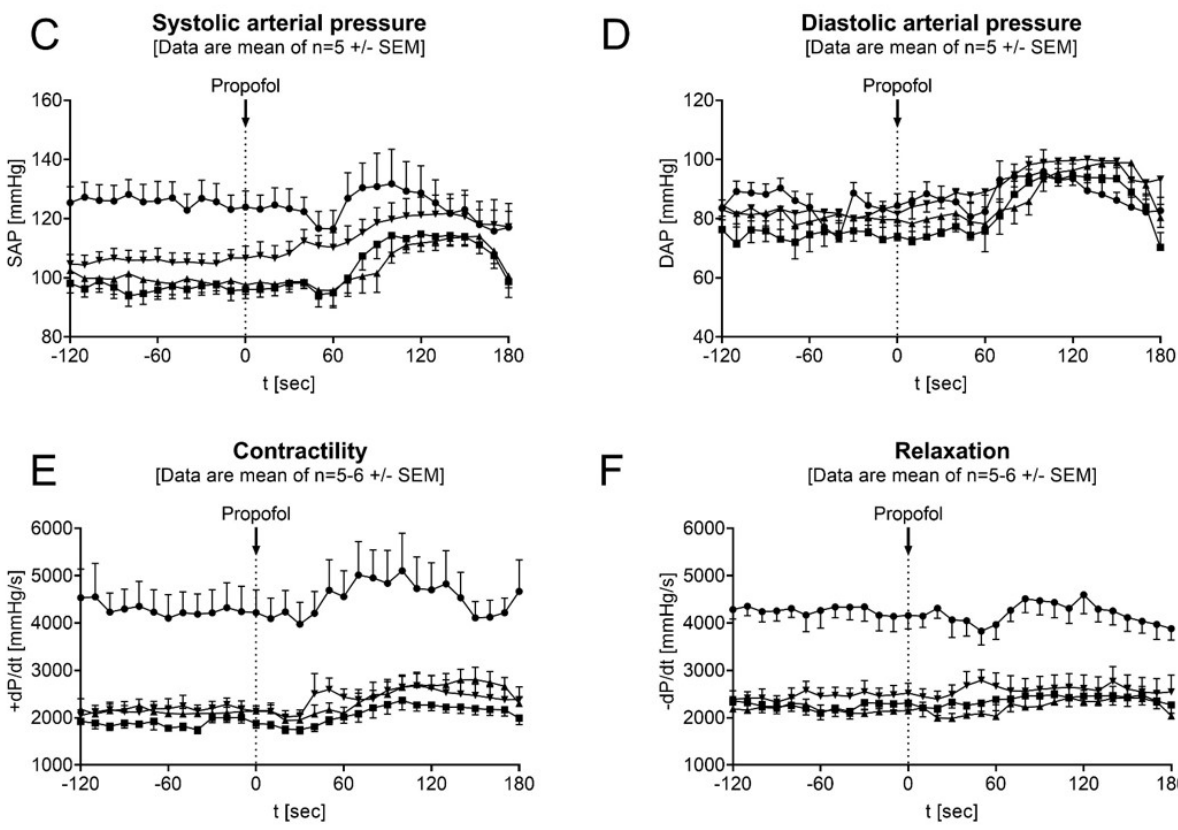

F
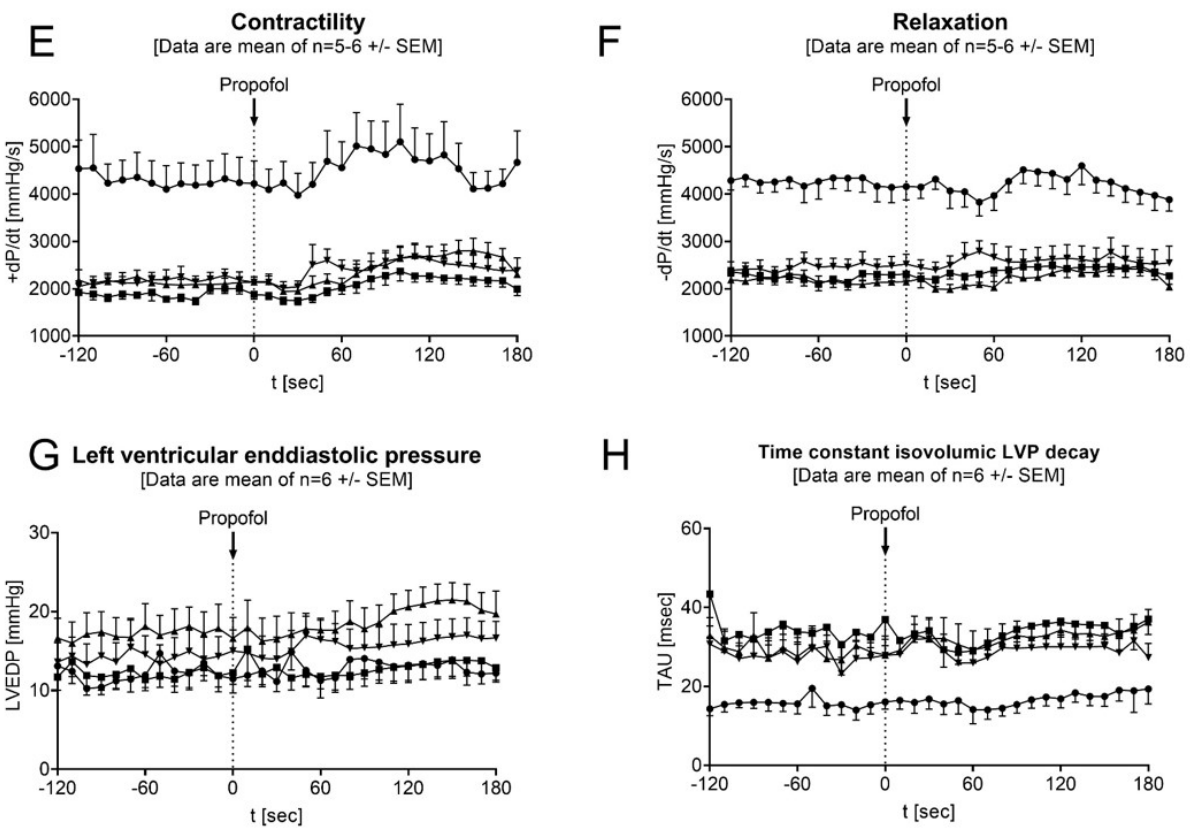

Figure 3. Continuous hemodynamic monitoring during anesthesia initiation with propofol. The different lines are representing the data recordings during healthy conditions days of pacing $(\boldsymbol{\square}), 28$ days of pacing $(\mathbf{\Delta})$ and 42 days of pacing $(\boldsymbol{\nabla})$ 
more pronounced as HF progressed (Figure 4G). At peak, an increase of $+4.3 \pm 3.9 \mathrm{mmHg}(\mathrm{p}=0.242)$ in LVEDP was observed $150 \mathrm{sec}$ after propofol in animals after they have been paced for 28 days, which was also the highest level for LVEDP with $21.5 \pm 5.3 \mathrm{mmHg}$ in the complete study. In Figure $3 \mathrm{H}$ and $4 \mathrm{H}$ the time constant of isovolumetric LVP decay (Tau) is shown. This marker represents a parameter of diastolic function. With the exception of a short-term increase $(+5.1 \pm 1.6 \mathrm{msec}$, $\mathrm{p}=0.0035)$ immediately after propofol injection $(20-30$ seconds post propofol, after 42 days of pacing, $\mathrm{p}<0.05)$ no significant effects were observed after bolus injection of propofol.
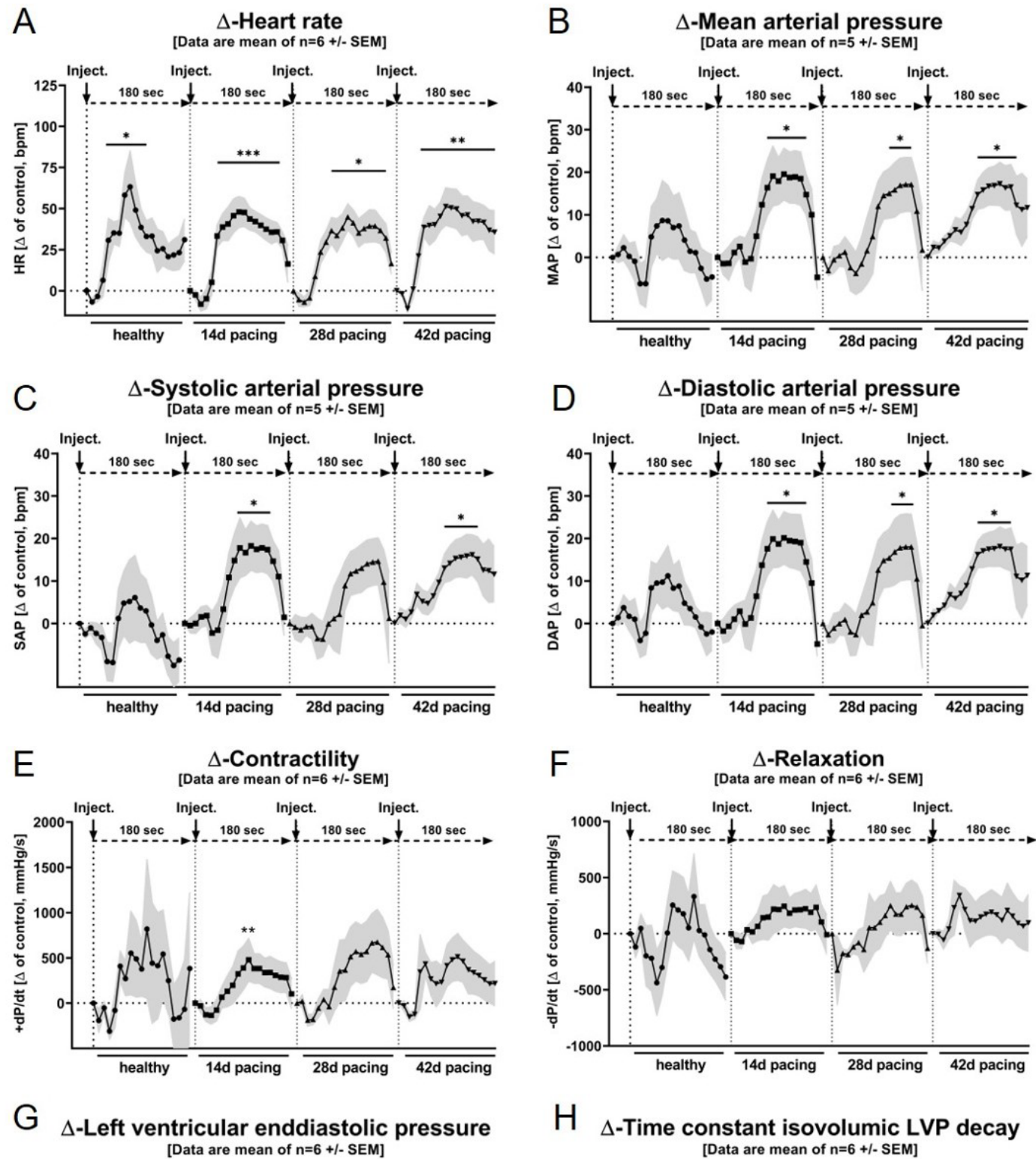

\section{H $\Delta$-Time constant isovolumic LVP decay}

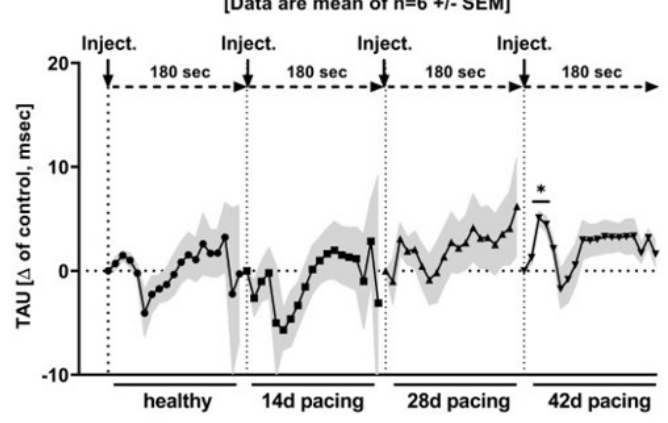

Figure 4. Changes to individual baseline values. Baseline hemodynamic data $(-120-0 \mathrm{sec})$ were averaged and compared to individual data post application $(0-180$ sec $)$. Data are represented as mean of $n=5-6$ animals + - SEM. Statistics were described in the text. The different lines are representing the data recordings during healthy conditions ( $)$, after 14 days of pacing $(\square), 28$ days of pacing $(\boldsymbol{\Delta})$ and 42 days of pacing $(\boldsymbol{\nabla})$ 


\section{Discussion}

There are several anesthetics available for cardiac and non-cardiac surgery that show a short half-live and a fast onset of action like etomidate and propofol. Etomidate is the drug of choice for the sedation of patients with risk for hypotension and being more frequently used than propofol in patients with heart failure, because of safety aspects $[18,19]$. Hemodynamic response after etomidate and propofol administration is partly modulated by changes in baroreceptor sensitivity. Arterial baroreceptors are control mechanisms that regulate arterial pressure by modifying myocardial contractility, $\mathrm{HR}$ and vascular resistance [20]. It is well known that etomidate has only slight effects on baroreceptors and therefore it is characterized by its hemodynamic stability. In contrast, propofol has an effect on that mentioned baroreflex control that leads to tachycardia and hypotension [21-23]. It is important to know that patients with HF have a higher incidence of post-induction hypotension [12], which makes propofol critical in this population. Until now, it is obvious that these differences make etomidate to the agent of choice for anesthesia in patients with cardiac dysfunction [24]. However, etomidate has some general side effects: e.g., due to postintervention nausea and vomiting etomidate is rarely used in patients undergoing gastroscopy [25], where propofol is used as an alternative [26,27].

There are some studies which describe a significant increase of morbidity and mortality in patients with HF undergoing surgery under general anesthesia because a reduction of arterial pressure during and after anesthesia [28]. This can lead to reduced end organ perfusion resulting in worsening of HF [26]. Less is known about the effect of propofol on cardiac and systemic function during immediate anesthesia initiation. However, with an increase of approximately $60 \%$ of cardiac device implantations (e.g. pacemaker and ICD's) in the US from 1997 to 2004 and the rising number of other cardiac procedures, like catheter ablations, arrhythmia cardioversions or transesophageal echocardiography, safety of short-term anesthesia plays an important role in patients with HF [29]. Since propofol is still an alternative in patients with $\mathrm{HF}$, it is of great interest to know the exact hemodynamics in all phases of anesthesia (including the initiation phase). Our study evaluated the safety and tolerability of anesthesia initiation with propofol in healthy versus HF subjects.

In our study we used the 'tachypacing-induced HF model', which is one of the commonly used animal models in cardiovascular research [30]. Characterized by dilated ventricles and a reduced ejection which makes it per definition to a HF with reduced ejection fraction (HFrEF) model. The advantage of this large animal model is a close-to-human anatomy and cardiac physiology, low variability in phenotype and patient-oriented characteristics. Echocardiography data, biomarker and hemodynamic recordings confirmed the expected left ventricular depression representing the development of an HF phenotype after several weeks of right ventricular pacing. The novelty of this model consisted in the use of a telemetric systems which allows for high-resolution recordings of cardiac and systemic hemodynamics continuously in conscious animals. It was also possible to analyze the disease progression and the induction of anesthesia without any gaps. Changes observed were an increase in heart rate and Tau, as well as a decrease in $+\mathrm{dP} / \mathrm{dt}$ and $-\mathrm{dP} / \mathrm{dt}$.

In our study we used a dose of $5 \mathrm{mg} / \mathrm{kg}$ bodyweight. This dose was chosen because it is referenced for short procedures, e.g. endotracheal intubation prior to a surgical intervention [31].
From the literature is known that long lasting propofol could lead to a depression of sympathetic activity and a reduced vascular resistance [32]. Interestingly, we observed only a slight drop in BP 60 seconds after application. This effect was most pronounced in healthy animals and was attenuated with increasing progression of the disease. After this drop in BP, we observed a robust and significant increase in $\mathrm{BP}$ in all HF-groups. It can be speculated that the increase in BP is triggered by the elevated HR and consequent increase in cardiac output. From previous investigations in patients it is known, that propofol infusion over a period of 30 minutes leads to a decrease in HR by resetting the baroreceptor reflex [20,33]. We demonstrate an increase in HR in healthy as well as in HF dogs. These effects are in accordance with previous preclinical studies, investigating the effect of anesthesia initiation with propofol, but are fundamentally different from observations during continuous infusion $[13,19,33]$. By analyzing the left ventricular pressure signal, we could not detect any changes in myocardial relaxation after propofol, whereas contractility shows a slight increase. This effect could be also primarily caused by the increase in heart rate.

Patients with reduced EF often suffer from fluid overload reflected in elevated left ventricular enddiastolic pressures (LVEDP). This parameter describes the filling pressure of the left ventricle and increased values implies a left ventricular dysfunction [34] and can be further associated with renal dysfunction [35]. Therefore, one therapeutic aim should be to protect patients from a drastic rise in LVEDP. In our study, we could observe only a slight increase after propofol bolus in HF-dogs.

The results of our study should be considered in the light of its limitations: By only studying six dogs, this study has a low number of test subjects. On the other side, this model shows a low degree of variability and experimental conditions were well controlled [15]. Second, by using the tachypacing-induced HF model, only a specific subpopulation of HF patients is represented in our study. In further studies, the influence of an anesthetic induction could also be tested in other in vivo models and thus in other phenotypes of HF. Also, we did not compare the propofol application head-to-head with other anesthetic agents. A possible next step could be the characterization of competing anesthetics in the same model to compare hemodynamic effects. Propofol showed the same directional effect in healthy and diseased animals and does not have a more pronounced influence on disturbed left ventricular conditions of the heart or leads to a further drop in BP.

\section{Author's individual contribution}

Julia Vogel: This author helped conceive the original idea; design the study; acquire the data; interpret and analyze the data; review the literature; write the first draft of the manuscript; and approve the final manuscript

Philip Boehme, M.D. PhD.: This author helped critically review the manuscript and approve the final manuscript.

Reza Wakili, M.D.: This author helped critically review the manuscript and approve the final manuscript.

Katharina Boden: This author helped critically review the manuscript and approve the final manuscript.

Thomas Reimer, M.D.: This author helped critically review the manuscript and approve the final manuscript. 
Peter Sandner, PhD.: This author helped critically review the manuscript and approve the final manuscript.

Jörg Hüser, PhD.: This author helped critically review the manuscript and approve the final manuscript.

Hubert Truebel, M.D.: This author helped critically review the manuscript and approve the final manuscript.

Thomas Mondritzki, PhD.: This author helped conceive the original idea; design the study; acquire the data; interpret and analyze the data; review the literature; write the first draft of the manuscript; and approve the final manuscript.

\section{Funding}

Funding was provided by Bayer AG.

\section{Declaration of conflicting interest}

At the time of the study was performed the following authors were employees of Bayer AG: JV, PB, KB, TR, PS, JH, WD, HT, TM.

\section{Findings}

Propofol showed the same directional effect in healthy and diseased animals and does not have a more pronounced influence on disturbed left ventricular conditions of the heart or leads to a further drop in blood pressure.

\section{Meaning}

In summary, this study could help to better understand hemodynamics of propofol and thereby help improve patient safety during anesthesia induction by unmasking intra-cardiac hemodynamics.

\section{References}

1. Ambrosy AP, Fonarow GC, Butler J, Chioncel O, Greene SJ, et al. (2014) The global health and economic burden of hospitalizations for heart failure: lessons learned from hospitalized heart failure registries. Journal of the American College of Cardiology 63: 1123-1133. [Crossref]

2. Hammill BG, Curtis LH, Bennett-Guerrero E, O'Connor CM, Jollis JG, et al. (2008) Impact of heart failure on patients undergoing major noncardiac surgery. Anesthesiology 108: $559-567$

3. Nadziakiewicz P, Pacholewicz J, Zakliczynski M, Niklewski T, Borkowski J, et al (2016) Comparison of Mechanical Circulatory Support by the Use of Pulsatile Left Ventricular Assist Devices Polvad MEV and Continuous Flow Heart Ware and Heart Mate II in a Single-Center Experience. Transplantation proceedings 48: 1770-1774.

4. Chen C-Q, Wang X, Zhang J, Zhu S-M (2017) Anesthetic management of patients with dilated cardiomyopathy for noncardiac surgery. European review for medical and pharmacological sciences 21: 627-634.

5. Ponikowski P, Voors AA, Anker SD, Bueno H, Cleland JGF, et al. (2016) ESC Guidelines for the diagnosis and treatment of acute and chronic heart failure: The Task Force for the diagnosis and treatment of acute and chronic heart failure of the European Society of Cardiology (ESC)Developed with the special contribution of the Heart Failure Association (HFA) of the ESC. European heart journal 37: 2129-200.

6. Rouby JJ, Andreev A, Léger P, Arthaud M, Landault C, et al. (1991) Peripheral vascular effects of thiopental and propofol in humans with artificial hearts. Anesthesiology 75: 32-42. [Crossref]

7. Hert SG de, Vermeyen KM, Adriaensen HF (1990) Influence of thiopental, etomidate, and propofol on regional myocardial function in the normal and acute ischemic heart segment in dogs. Anesthesia and analgesia 70: 600-607.

8. Blake DW, Way D, Trigg L, McGrath BP (1994) Regional blood flow effects of dopexamine versus enalaprilat during propofol anaesthesia in rabbits with experimental chronic heart failure. Cardiovascular research 28: 710-714.

9. Hebbar L, Dorman BH, Clair MJ, Roy RC, Spinale FG, et al. (1997) Negative and selective effects of propofol on isolated swine myocyte contractile function in pacinginduced congestive heart failure. Anesthesiology 86: 649-659.
10. Aghdaii N, Ziyaeifard M, Faritus SZ, Azarfarin R (2015) Hemodynamic Responses to Two Different Anesthesia Regimens in Compromised Left Ventricular Function Patients Undergoing Coronary Artery Bypass Graft Surgery: Etomidate-Midazolam Versus Propofol-Ketamine. Anesthesiology and pain medicine 5: e27966.

11. Glen JB (1980) Animal studies of the anaesthetic activity of ICI 35 868. British journal of anaesthesia 52 : 731-742.

12. Baradari AG, Alipour A, Habibi MR, Rashidaei S, Emami Zeydi A, et al. (2017) A randomized clinical trial comparing hemodynamic responses to ketamine-propofol combination (ketofol) versus etomidate during anesthesia induction in patients with left ventricular dysfunction undergoing coronary artery bypass graft surgery. Archives of medical science 13: 1102-1110. [Crossref]

13. Mather LE, Duke CC, Ladd LA, Copeland SE, Gallagher G, et al. (2004) Direct cardiac effects of coronary site-directed thiopental and its enantiomers: a comparison to propofol in conscious sheep. Anesthesiology 101: 354-364.

14. Kong W, Huang J, Rollins DL, Ideker RE, Smith WM, et al. (2007) A semiimplantable multichannel telemetry system for continuous electrical, mechanical and hemodynamical recordings in animal cardiac research. Physiological measurement 28 : 249-257.

15. Mondritzki T, Boehme P, White J, Park JW, Hoffmann J, et al. (2018) Remote Left Ventricular Hemodynamic Monitoring Using a Novel Intracardiac Sensor. Circulation. Cardiovascular interventions 11: e006258.

16. Mondritzki T, Boehme P, Schramm L, Vogel J, Mathar I, et al. (2018) New pulmonary hypertension model in conscious dogs to investigate pulmonary-selectivity of acute pharmacological interventions. European journal of applied physiology 118: 195-203.

17. Yancy CW, Jessup M, Bozkurt B, Butler J, Casey DE, et al. (2017) ACC/AHA/HFSA Focused Update of the 2013 ACCF/AHA Guideline for the Management of Heart Failure: A Report of the American College of Cardiology/American Heart Association Task Force on Clinical Practice Guidelines and the Heart Failure Society of America Circulation 136: e137-e161.

18. Dhillon A, Khanna A, Randhawa MS, Cywinski J, Saager L, et al. (2016) Perioperative outcomes of patients with hypertrophic cardiomyopathy undergoing non-cardiac surgery. Heart (British Cardiac Society) 102: 1627-1632.

19. Yuki K, Lee S, Staffa SJ, DiNardo JA (2018) Induction techniques for pediatric patients with congenital heart disease undergoing noncardiac procedures are influenced by cardiac functional status and residual lesion burden. Journal of clinical anesthesia 50 14-17. [Crossref]

20. Cullen PM, Turtle M, Prys-Roberts C, Way WL, Dye J (1987) Effect of propofo anesthesia on baroreflex activity in humans. Anesthesia and analgesia 66: 1115-1120.

21. Florea VG, Cohn JN (2014) The autonomic nervous system and heart failure. Circulation research 114: 1815-1826.

22. Ebert TJ, Muzi M (1994) Propofol and autonomic reflex function in humans. Anesthesia and analgesia 78 : 369-375.

23. Grounds RM, Twigley AJ, Carli F, Whitwam JG, Morgan M, et al. (1985) The haemodynamic effects of intravenous induction. Comparison of the effects of thiopentone and propofol. Anaesthesia 40: 735-740.

24. Aggarwal S, Goyal VK, Chaturvedi SK, Mathur V, Baj B, et al. (2016) A comparative study between propofol and etomidate in patients under general anesthesia. Brazilian journal of anesthesiology (Elsevier) 66: 237-241.

25. Meng Q-T, Cao C, Liu H-M, Xia Z-Y, Li W, et al. (2016) Safety and efficacy of etomidate and propofol anesthesia in elderly patients undergoing gastroscopy: A double-blind randomized clinical study. Experimental and therapeutic medicine 12 $1515-1524$.

26. Toman H, Erkılinc A, Kocak T, Guzelmeric F, Savluk OF, et al. (2016) Sedation for transesophageal echocardiography: comparison of propofol, midazolam and midazolam-alfentanil combination. Medicinski glasnik: official publication of the Medical Association of Zenica-Doboj Canton, Bosnia and Herzegovina 13: 18-24. [Crossref]

27. Wang D, Chen C, Chen J, Xu Y, Wang L, et al. (2013) The use of propofol as a sedative agent in gastrointestinal endoscopy: a meta-analysis. PloS one 8: e53311.

28. Roshanov PS, Sheth T, Duceppe E, Tandon V, Bessissow A, et al. (2019) Relationship between Perioperative Hypotension and Perioperative Cardiovascular Events in Patients with Coronary Artery Disease Undergoing Major Noncardiac Surgery. Anesthesiology 130: 756-766. 
29. Zhan C, Baine WB, Sedrakyan A, Steiner C (2008) Cardiac device implantation in the United States from 1997 through 2004: a population-based analysis. Journal of general internal medicine 23: 13-19. [Crossref]

30. Powers JC, Recchia F (2018) Canine Model of Pacing-Induced Heart Failure. Methods in molecular biology (Clifton, N.J.) 1816: 309-325.

31. Glowaski MM, Wetmore LA (1999) Propofol: application in veterinary sedation and anesthesia. Clinical techniques in small animal practice 14: 1-9.

32. Singh R, Choudhury M, Kapoor PM, Kiran U (2010) A randomized trial of anesthetic induction agents in patients with coronary artery disease and left ventricular dysfunction. Annals of cardiac anaesthesia 13: 217-223.
33. Pernerstorfer T, Mayer N, Jilma B, Krenn CG, Bayer GS, et al. (1997) Does phosphodiesterase III inhibition reverse the cardiodepressive effects of propofol? Anesthesia and analgesia 85: 725-728.

34. Vasan RS, Levy D (2000) Defining diastolic heart failure: a call for standardized diagnostic criteria. Circulation 101: 2118-2121.

35. Grodin JL, Drazner MH, Dupont M, Mullens W, Taylor DO, et al. (2015) A disproportionate elevation in right ventricular filling pressure, in relation to left ventricular filling pressure, is associated with renal impairment and increased mortality in advanced decompensated heart failure. American heart journal 169: 806-812. [Crossref]

Copyright: (C2020 Vogel J. This is an open-access article distributed under the terms of the Creative Commons Attribution License, which permits unrestricted use, distribution, and reproduction in any medium, provided the original author and source are credited. 